\title{
XMM-Newton observations of the Small Magellanic Cloud: XMMU J004814.1-731003, a 25.55 s Be/X-ray binary pulsar ${ }^{\star}$
}

\author{
F. Haberl ${ }^{1}$, P. Eger ${ }^{1}$, W. Pietsch ${ }^{1}$, R. H. D. Corbet $^{2}$, and M. Sasaki ${ }^{1}$
}

\author{
1 Max-Planck-Institut für extraterrestrische Physik, Giessenbachstraße, 85748 Garching, Germany \\ e-mail: fwh@mpe.mpg.de \\ 2 University of Maryland, Baltimore County; X-ray Astrophysics Laboratory, Mail Code 662, NASA Goddard Space Flight Center, \\ Greenbelt, MD 20771, USA
}

Received 8 February 2008 / Accepted 6 March 2008

\section{ABSTRACT}

\begin{abstract}
Aims. To investigate candidates for Be/X-ray binaries in the Small Magellanic Cloud (SMC), we observed a region around the emission nebula N19 with XMM-Newton in October 2006.

Methods. We analysed the EPIC data of the detected point sources to derive their spectral and temporal characteristics.

Results. We detected X-ray pulsations with a period of 25.550(2) s from the second-brightest source in the field, which we designate XMMU J004814.1-731003. The X-ray spectrum is well modelled by a highly absorbed $\left(N_{\mathrm{H}}=5 \times 10^{22} \mathrm{~cm}^{-2}\right)$ power-law with photon index $1.33 \pm 0.27$. The precise X-ray position allows us to identify a Be star as the optical counterpart. XMMU J004814.1-731003 is located within the error circle of the transient ASCA source AX J0048.2-7309, but its position is inconsistent with that of the proposed optical counterpart of AX J0048.2-7309 (the emission line star [MA93] 215). It remains unclear if XMMU J004814.1-731003 is associated with AX J0048.2-7309. XMMU J004814.1-731003 might be identical to an RXTE pulsar that was discovered with a period of $25.5 \mathrm{~s}$, but which is listed as a $51 \mathrm{~s}$ pulsar in the recent literature.
\end{abstract}

Key words. galaxies: individual: small Magellanic Cloud - galaxy: stellar content - stars: emission-line, Be - stars: neutron X-rays: binaries

\section{Introduction}

The region around the emission nebula N19 in the southwestern part of the SMC shows complex X-ray emission from several supernova remnants and high mass X-ray binaries (HMXBs). Several X-ray pulsars which are most likely associated with $\mathrm{Be} / \mathrm{X}$-ray binaries, the major subclass of HMXBs, were detected with ASCA and RXTE, but could not be accurately located and therefore, lack a clear optical identification. Southeast of N19, ASCA detected a $9.1 \mathrm{~s}$ pulsar (Ueno et al. 2000) where ROSAT PSPC images show two close hard X-ray sources, both possibly contributing to the ASCA source (Filipović et al. 2000). Both these objects are most likely $\mathrm{Be} / \mathrm{X}$-ray binaries and it is not clear which is the $9.1 \mathrm{~s}$ pulsar (Coe et al. 2005, and references therein). Another nearby ASCA source (AX J0048.2-7309) was classified as candidate $\mathrm{Be} / \mathrm{X}$-ray binary by Yokogawa et al. (2003) from its X-ray spectral properties. In the error circle of AX J0048.2-7309 the $\mathrm{H} \alpha$ emission line star [MA93]215 (Meyssonnier \& Azzopardi 1993) is located, suggesting an association with a Be star. However, this requires confirmation from an improved X-ray position. ROSAT did not detect any source at the ASCA position but a faint source was seen during an early XMM-Newton observation in August 2002 (Shtykovskiy \& Gilfanov 2005). RXTE discovered pulsations with periods of $16.6 \mathrm{~s}$ and $25.5 \mathrm{~s}$ during an observation in September 2000, viewing an area near the southwestern edge of the SMC (Lamb et al. 2002). The pulse period from the latter source was later argued

\footnotetext{
* Based on observations with XMM-Newton, an ESA Science Mission with instruments and contributions directly funded by ESA Member states and the USA (NASA).
}

to be the harmonic of a $51 \mathrm{~s}$ spin period (Laycock et al. 2005). Neither pulsars could ever be better localised or optically identified.

In the course of our AO5 XMM-Newton program to investigate candidates for HMXBs in the SMC, we observed the region around N19. The XMM-Newton source detected by Shtykovskiy \& Gilfanov (2005) was seen again and we analysed the EPIC data to investigate the nature of this object, designated XMMU J004814.1-731003. Here we report on results from a temporal and spectral analysis of the X-ray data of XMMU J004814.1-731003 and identify a Be star as the optical counterpart. This adds XMMU J004814.1-731003 to the numerous Be/X-ray binary pulsars known in the SMC (for recent reviews see Haberl \& Pietsch 2004; Coe et al. 2005).

\section{Data analysis and results}

We observed the field around the emission nebula N19 with XMM-Newton (Jansen et al. 2001) on 2006 October 5. The EPIC-MOS (Turner et al. 2001) and EPIC-PN (Strüder et al. 2001) cameras were operated in imaging mode (see Table 1). For the X-ray analysis we used the XMM-Newton Science Analysis System (SAS) version 7.1.0 supported by tools from the FTOOLS package together with XSPEC version 11.3.2p for spectral modelling.

A source, not seen with ROSAT was detected near the centre of the field of view and inside the error circle of the ASCA source AX J0048.2-7309 (Yokogawa et al. 2003). After astrometric boresight correction (see also Haberl \& Pietsch 2008) we determined the position of the source to be RA $=004814.10$ 
Table 1. Details of the XMM-Newton EPIC observation.

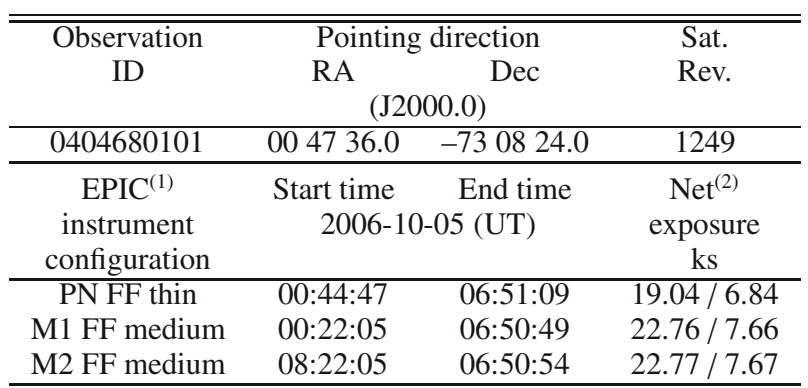

(1) FF: full frame CCD readout mode with $73 \mathrm{~ms}$ frame time for PN and $2.6 \mathrm{~s}$ for MOS; thin and medium optical blocking filters.

(2) Left: full exposure times as used for spectral and temporal analysis. Right: Exposure after removing intervals of high background (used for source detection analysis, see text).

and Dec $=-731004.0$ (J2000.0) using the SAS standard maximum likelihood technique for source detection and assign the name XMMU J004814.1-731003 (hereafter J0048). The $1 \sigma$ position error is $1.2^{\prime \prime}$, dominated by the remaining systematic uncertainty of $1.1^{\prime \prime}$. Due to background flaring activity during part of the observation we applied a background screening before our source detection analysis, but used the full exposure times for the extraction of light curves and spectra of individual sources. The resulting net exposures are listed in Table 1.

The precise X-ray position inferred from the EPIC data allowed us to identify the optical counterpart of J0048. The source position is incompatible with the emission line star [MA93] 215 (46" away from the X-ray position) which was suggested to be the optical counterpart of AX J0048.2-7309, but another star with optical properties of a B star is found at the XMM-Newton $\mathrm{X}$-ray position (Fig. 1). In Table 2 optical brightness and colours taken from the UBVR CCD Survey of the Magellanic Clouds (Massey 2002), the Magellanic Clouds Photometric Survey (MCPS, Zaritsky et al. 2002) and the OGLE BVI photometry catalogue (Udalski et al. 1998) are given.

To investigate longterm brightness variations of the optical counterpart we retrieved light curves in the $I$-band from the OGLE photometry database (star 171264; Szymanski 2005; Udalski et al. 1997) and the $B$ - and $R$-band from the MACHO survey (star 212.15849.52). The light curves in all bands show a gradual fading over the total observing period with additional variations of $\sim 0.2$ mag superimposed (Fig. 2). We applied an FFT analysis to the MACHO $R$ - and $B$-band data with a magnitude error < $0.05 \mathrm{mag}$ (Lomb 1976; Scargle 1982) to determine whether these changes are periodic. We investigated the period range between 1 day and 1000 days which revealed peaks at short and long periods (Fig. 3). Peaks at 1.49 days, 1.81 days, 3.02 days and 5.93 days indicate similar periods as seen from other SMC Be/X-ray binaries (Schmidtke et al. 2004), but are probably too short for orbital periods. Broad peaks at long periods of a few hundred days ( $\sim 220$ days, $\sim 340$ days and $\sim 400$ days, $\sim 440$ days and $\sim 680$ days) indicate variations which are not strictly periodic.

The broad band X-ray light curve of J0048 indicates some intensity variations of the flux on timescales of $1-2 \mathrm{~h}$. But due to the low count rate (on average $\sim 0.03 \mathrm{cts} \mathrm{s}^{-1}$ in the EPIC-PN) the statistics are too poor to allow definite conclusions to be reached. We searched for X-ray pulsations in the EPIC data of J0048, after correcting the photon arrival times to the solar

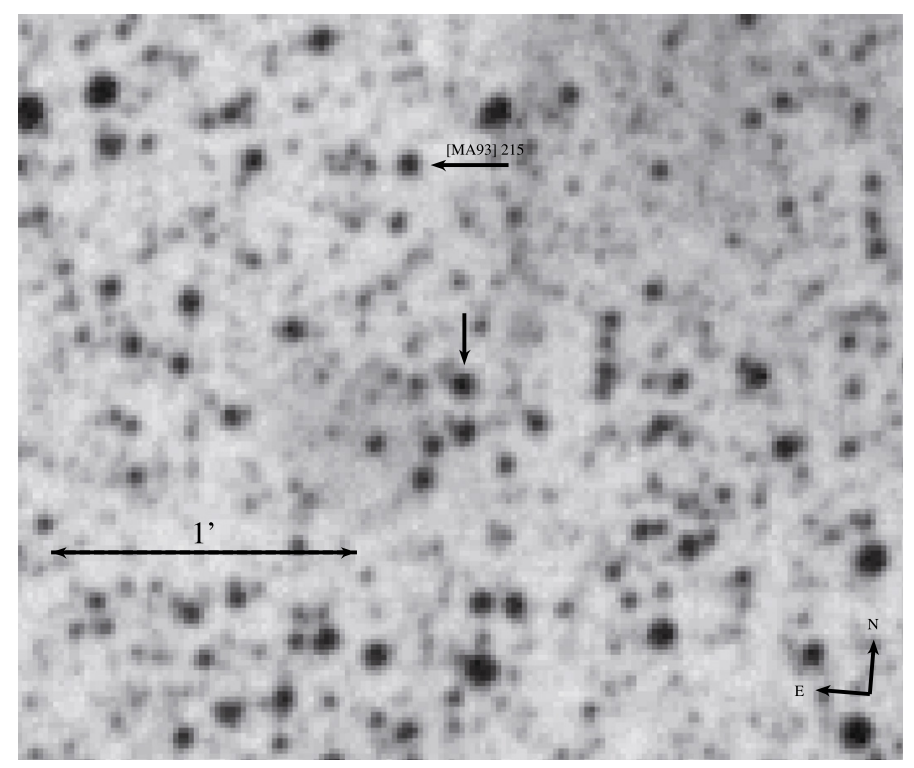

Fig. 1. Finding chart based on the DSS2 red image of the region around XMMU J004814.1-731003. The optical counterpart is marked with a vertical arrow. The error circle of the X-ray position is smaller than the size of the star on the image. The $\mathrm{H} \alpha$ emission line star [MA93] 215 is also labelled.

system barycentre. The Fourier power spectra obtained from the EPIC-PN data in different energy bands revealed a peak at $25.5 \mathrm{~s}$ which is most significant when selecting energies above $1 \mathrm{keV}$. For the band $1.0-10.0 \mathrm{keV}$ a power of $\sim 40$ is obtained. To verify the period, the MOS data were also investigated. Peaks at the same frequency were present for the individual MOS light curves, but not significant on their own. Finally, combining the events from the two MOS cameras also resulted in the highest peak at the same frequency. Combining all EPIC data yields a maximum power of $\sim 60$ (Fig. 4). Although no significant power around $51 \mathrm{~s}$ is seen in the power spectrum, we investigated this period range using the Rayleigh $Z^{2}$ method (Buccheri et al. 1983) with fundamental and one harmonic frequency involved. The resulting maximum $Z^{2}$ power is 69.5 with $Z_{1}^{2}=2.9$ for the fundamental (at $51.1 \mathrm{~s}$ ) and $Z_{2}^{2}=66.6$ for the first harmonic (at $25.55 \mathrm{~s}$ ). We conclude that there is no significant modulation with a period of $51 \mathrm{~s}$. To derive an accurate value and error for the detected pulse period we used the Bayesian method (Gregory \& Loredo 1996) as described in Zavlin et al. (2000). The pulse period is determined to $25.550 \pm 0.002 \mathrm{~s}$ ( $1 \sigma$ error).

We folded the X-ray light curves on the best period in the standard EPIC energy bands $(0.2-0.5 \mathrm{keV}, 0.5-1.0 \mathrm{keV}, 1.0-$ $2.0 \mathrm{keV}, 2.0-4.5 \mathrm{keV}, 4.5-10.0 \mathrm{keV}$ and the broad band $0.2-$ $10.0 \mathrm{keV}$ ) and show the resulting pulse profiles in Fig. 5. The pulse profile of J0048 is dominated by a broad main pulse which varies in shape with energy. At energies below $1 \mathrm{keV}$ the high level of absorption (see below) strongly reduces the count rate and a modulation is only marginally seen. The pulsed fraction (derived from modelling the pulse profile with two sine waves) in the total energy band is $(60 \pm 20) \%$. There is some indication for a higher pulsed fraction at energies below $1 \mathrm{keV}$ and above $4.5 \mathrm{keV}$, but the number of counts in the individual bands is insufficient for this to reach statistical significance.

For the analysis of the X-ray spectra we extracted pulsephase averaged EPIC spectra for PN (single + double pixel events, PATTERN 0-4) and MOS (PATTERN 0-12) 
Table 2. Optical identification.

\begin{tabular}{llcccccc}
\hline \hline Source & Catalogue & RA and Dec (J2000.0) & $V$ mag & $B-V$ & $U-B$ & $V-R$ & $V-I$ \\
\hline XMMU J004814.1-731003 & UBVR & $004814.10-731004.0$ & 15.25 & +0.13 & -0.64 & +0.12 & - \\
& MCPS & $004814.18-731003.9$ & 15.30 & +0.26 & -0.50 & - & -0.21 \\
& OGLE & $004814.13-731003.5$ & 15.71 & +0.01 & - & - & +0.09 \\
\hline
\end{tabular}

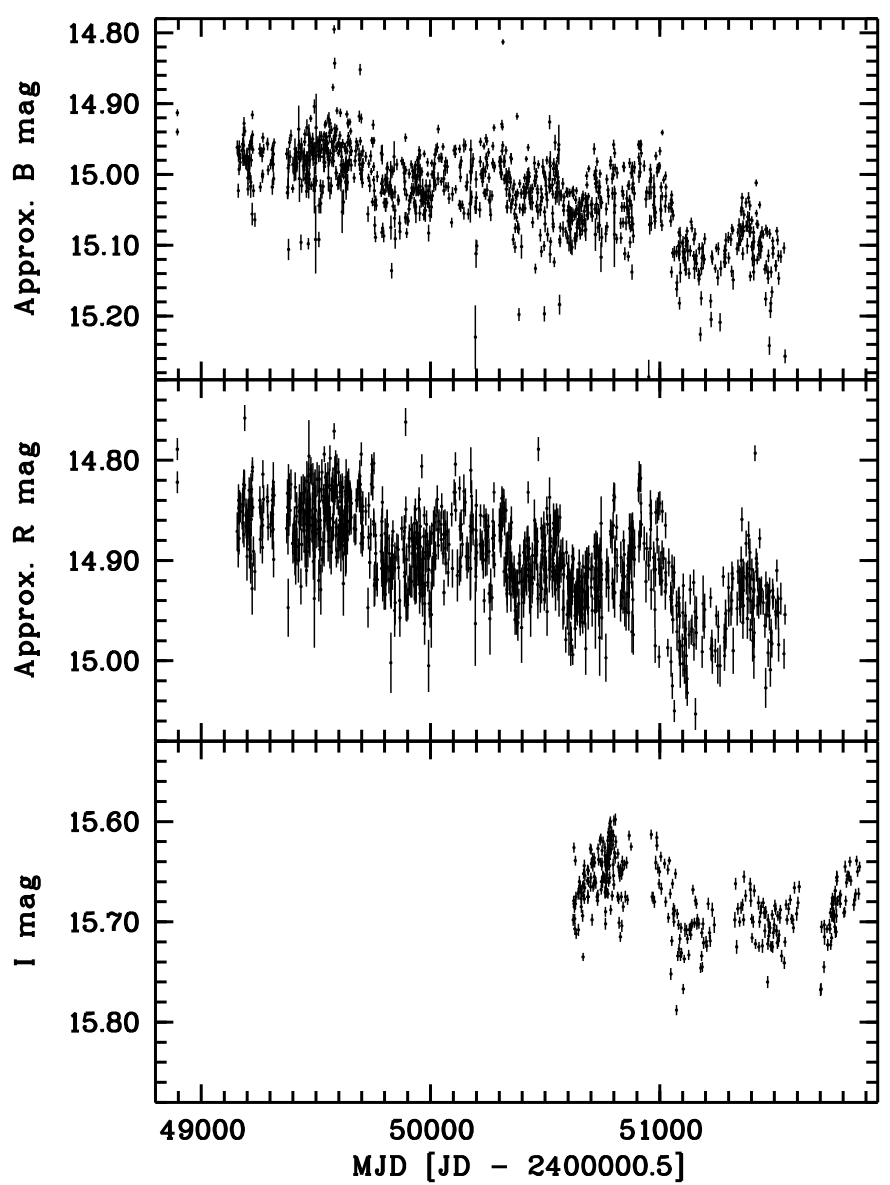

Fig. 2. MACHO $B$ - and $R$ - and OGLE $I$-band light curves of the optical counterpart of XMMU J004814.1-731003. For the MACHO data only data points with errors less than 0.05 mag are plotted.

disregarding bad CCD pixels and columns (FLAG 0). The three EPIC spectra were simultaneously fit with an absorbed powerlaw model allowing for a constant normalisation factor between the spectra. We used two absorption components, accounting for the Galactic foreground absorption (with a fixed hydrogen column density of $6 \times 10^{20} \mathrm{~cm}^{-2}$ and elemental abundances from Wilms et al. 2000) and the SMC absorption (with column density as a free parameter in the fit and with metal abundances reduced to 0.2 as typical for the SMC; Russell \& Dopita 1992). The best-fit (reduced $\chi^{2}=0.82$ for 27 degrees of freedom) power-law model yields a high absorption with $N_{\mathrm{H}}=$ $(5.2 \pm 2.0) \times 10^{22} \mathrm{~cm}^{-2}$, a photon index of $1.33 \pm 0.27$, an observed flux of $3.5 \times 10^{-13} \mathrm{erg} \mathrm{cm}^{-2} \mathrm{~s}^{-1}$ and a source luminosity of $2.1 \times 10^{35} \mathrm{erg} \mathrm{s}^{-1}(0.2-10.0 \mathrm{keV}$, assumed distance to the SMC of $60 \mathrm{kpc}$ Hilditch et al. 2005). Flux and luminosity refer to the values derived from the EPIC-PN spectrum, MOS1 and MOS2 yield somewhat lower values by $6 \%$ and $3 \%$, respectively. Errors for spectral parameters denote

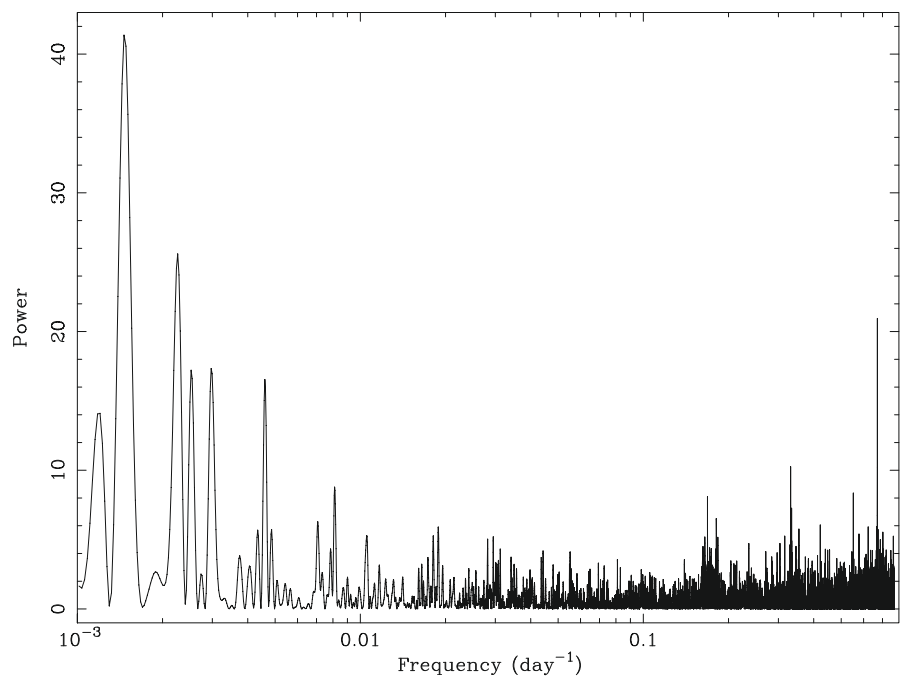

Fig. 3. Power spectrum of the MACHO $R$-band data of the optical counterpart (star 212.15849.52).

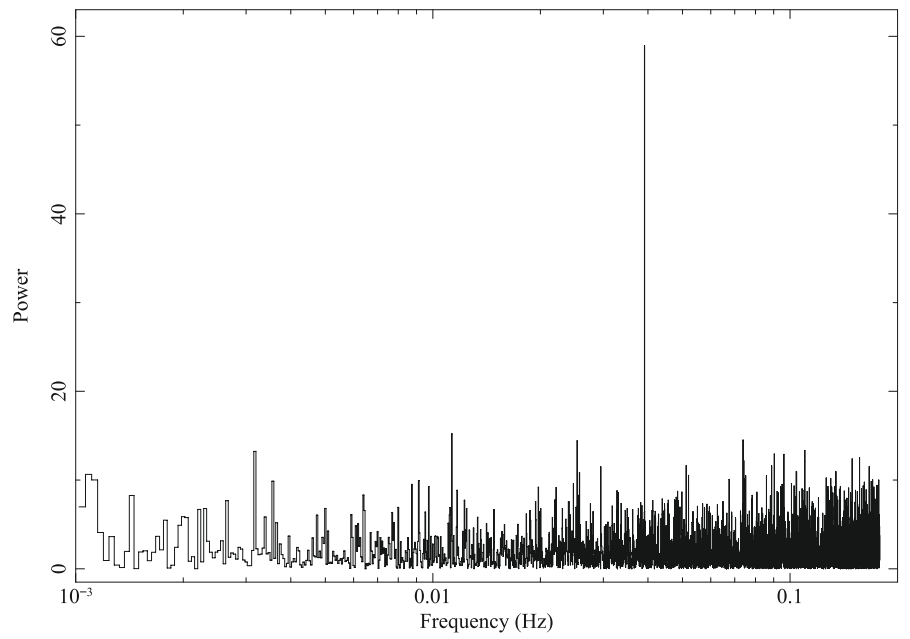

Fig. 4. Power spectrum of XMMU J004814.1-731003 produced from the $1.0-10.0 \mathrm{keV}$ combined EPIC data.

90\% confidence limits. The EPIC spectra and the best-fit model are shown in Fig. 6.

\section{Discussion}

We report the discovery of pulsations in the X-ray flux of J0048 with a period of $25.550 \mathrm{~s}$ and propose as the optical counterpart a $V \sim 15.5$ mag star. The X-ray spectrum of J0048 is well represented by a simple power-law, typical for Be/X-ray binary pulsars. However, the high absorption and the relative faintness of the source (the flux of $3.5 \times 10^{-13} \mathrm{erg} \mathrm{cm}^{-2} \mathrm{~s}^{-1}$ corresponds 


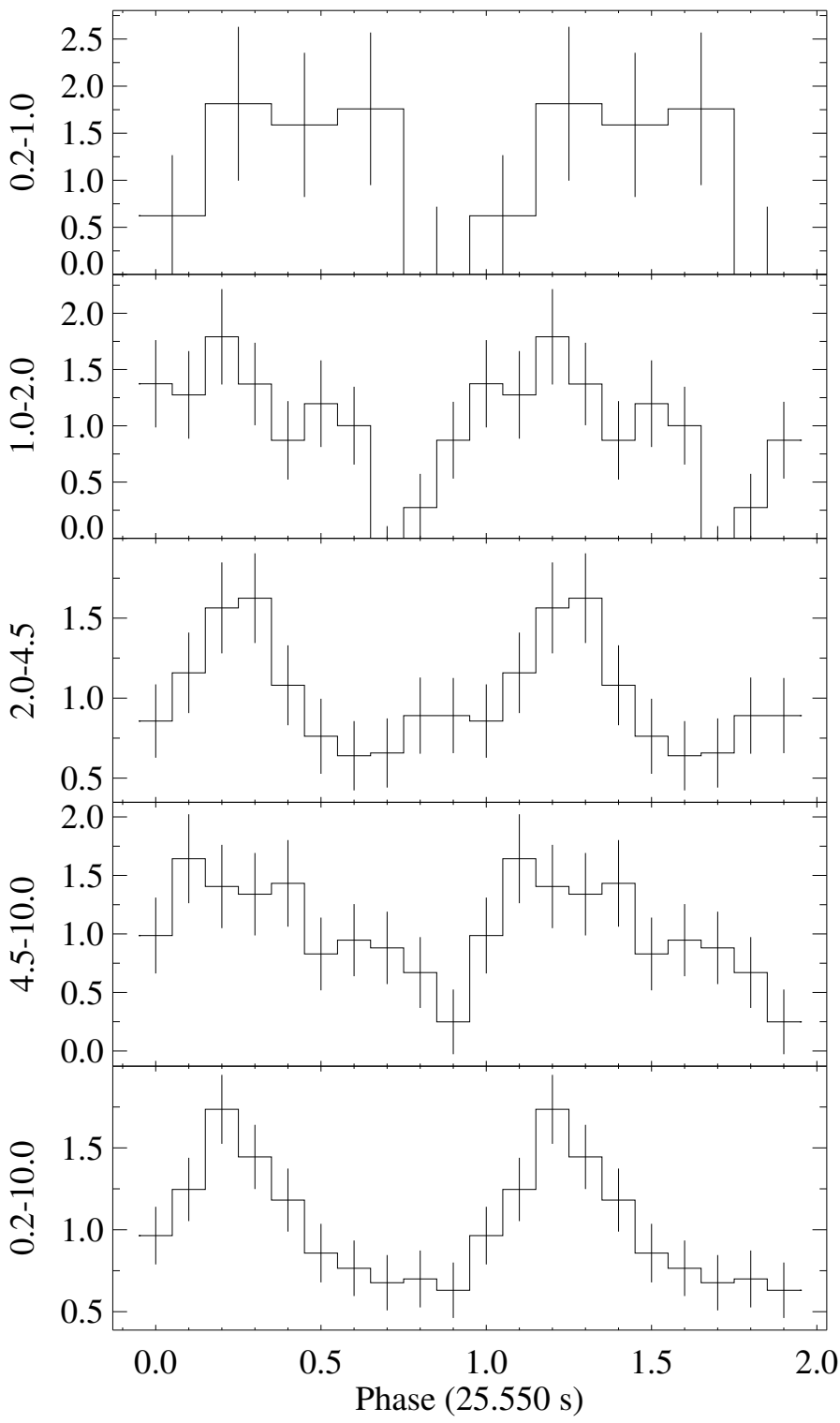

Fig. 5. Folded EPIC-PN light curves of XMMU J004814.1-731003 in the standard EPIC energy bands (the lower energy bands $0.2-0.5 \mathrm{keV}$ and $0.5-1.0 \mathrm{keV}$ were combined and rebinned by a factor of two due to the low count rate). The panels show the pulse profiles for the different energies specified in $\mathrm{keV}$. The intensity profiles are background subtracted and normalised to the average count rate (in cts s$~^{-1}: 0.00082$, $0.0073,0.0135,0.0093,0.0310$ from top to bottom).

to a source luminosity of $2.1 \times 10^{35} \mathrm{erg} \mathrm{s}^{-1}$ at the distance of the $\mathrm{SMC}$ ) prevent a more detailed spectral analysis.

Following Coe et al. (2005), we estimate the spectral class of the optical counterpart from its $B-V$ colour index to be B3 or later, depending on the observed $B-V$ index which is in the range 0.01 to 0.26 (Table 2 ). This assumes an extinction correction to the SMC of $E(B-V)=0.08$ (Schwering \& Israel 1991) and an additional correction of $(B-V)=-0.13$ to account for the presence of a circumstellar disc (Coe et al. 2005). However, in the Galaxy no Be/X-ray binaries with spectral type later than B2 are found (Negueruela 1998) which may indicate that this additional correction for J0048 is insufficient. This is supported by the large absorption seen in the X-ray spectrum of the pulsar. The significantly different values measured for $B-V$ in the different photometric surveys also suggest, that the extinction varies

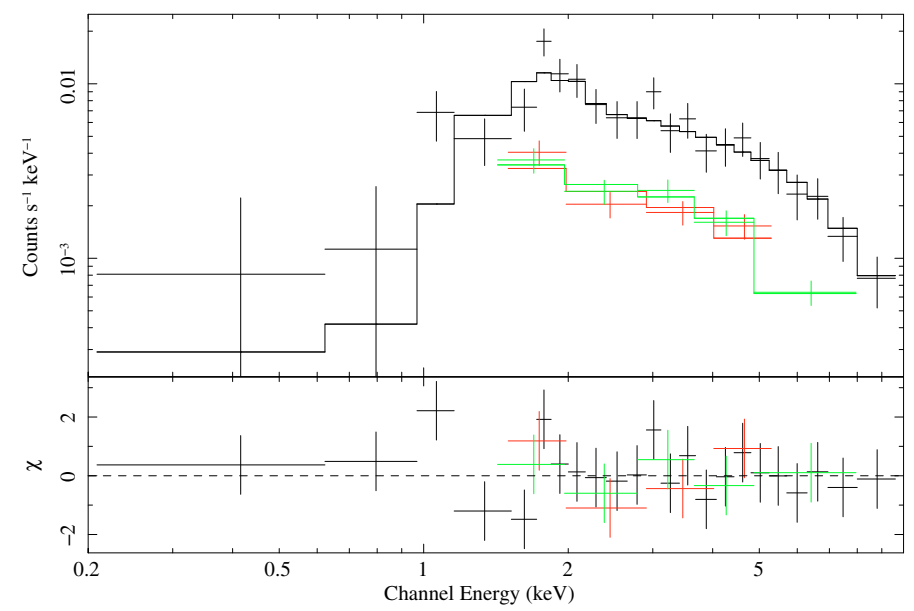

Fig. 6. EPIC spectra of XMMU J004814.1-731003. EPIC-PN is shown in black and EPIC-MOS in red (M1) and green (M2) (both grey in black and white representation). The histograms show the best-fit absorbed power-law model.

with time. In addition, a $B-R$ colour index derived from the approximate $B$ and $R$ light curves in Fig. 2 slowly increased after the end of 1998. At least this variable extinction must originate from matter local to the binary system and is most likely due to changes in the disc of the Be star.

From the relation between spin and orbital period (for a recent version of the "Corbet" diagram of SMC pulsars see Schmidtke \& Cowley 2005), we expect an orbital period between $\sim 25$ days and $\sim 150$ days for J0048. The optical light curves indicate various periodicities, which are either shorter or longer than this period range. Short periods between 3 and 11 days were also seen in other SMC Be/X-ray binaries and might be caused by a changing view of the Be disk region that is brightened by interaction with the neutron star Schmidtke et al. (2004). The star shows optical brightness variations on long time scales of a few hundred days which most likely are also associated with the Be star phenomenon, similar to, for example, XTE J0103-728 (=SXP6.85; McGowan et al. 2008), but with smaller amplitude.

The optical and X-ray properties identify J0048 as Be/X-ray binary pulsar in the SMC. The XMM-Newton source position is located within the error circle of AX J0048.2-7309, but the presence of another Be star ([MA93]215), which might be associated with AX J0048.2-7309, leaves it unclear if the ASCA source is identical with J0048. Only a retrospective detection of the pulse period in the ASCA data would allow an unambiguous identification. The pulsar might be identical to the $25.55 \mathrm{~s}$ pulsar seen by RXTE in September 2000 (Lamb et al. 2002). The RXTE observation was pointed at RA $=005044.64$ and Dec $=-731604.8$ which is $12^{\prime}$ away from J0048. Therefore J0048 was well within the RXTE PCA field of view of $1^{\circ}$ FWHM. However, in more recent literature (Laycock et al. 2005; Coe et al. 2005; Galache et al. 2008) the RXTE pulsar is listed with a period of $51 \mathrm{~s}$. Therefore, we can not exclude the possibility that the XMM-Newton and RXTE sources are two pulsars with different periods.

Acknowledgements. The XMM-Newton project is supported by the Bundesministerium für Wirtschaft und Technologie/Deutsches Zentrum für Luft- und Raumfahrt (BMWI/DLR, FKZ 50 OX 0001) and the Max-Planck Society. The "Second Epoch Survey" of the southern sky was produced by the Anglo-Australian Observatory (AAO) using the UK Schmidt Telescope. Plates from this survey have been digitised and compressed by the ST ScI. 
Produced under Contract No. NAS 5-26555 with the National Aeronautics and Space Administration. This paper utilises public domain data obtained by the MACHO Project, jointly funded by the US Department of Energy through the University of California, Lawrence Livermore National Laboratory under contract No. W-7405-Eng-48, by the National Science Foundation through the Center for Particle Astrophysics of the University of California under cooperative agreement AST-8809616, and by the Mount Stromlo and Siding Spring Observatory, part of the Australian National University.

\section{References}

Buccheri, R., Bennett, K., Bignami, G. F., et al. 1983, A\&A, 128, 245

Coe, M. J., Edge, W. R. T., Galache, J. L., \& McBride, V. A. 2005, MNRAS, 356,502

Filipović, M. D., Pietsch, W., \& Haberl, F. 2000, A\&A, 361, 823

Galache, J. L., Corbet, R. H. D., Coe, M. J., et al. 2008, ApJS, in press [arXiv:0802.2118]

Gregory, P. C., \& Loredo, T. J. 1996, ApJ, 473, 1059

Haberl, F., \& Pietsch, W. 2004, A\&A, 414, 667

Haberl, F., \& Pietsch, W. 2008, A\&A, 484, 451

Hilditch, R. W., Howarth, I. D., \& Harries, T. J. 2005, MNRAS, 357, 304

Jansen, F., Lumb, D., Altieri, B., et al. 2001, A\&A, 365, L1

Lamb, R. C., Macomb, D. J., Prince, T. A., \& Majid, W. A. 2002, ApJ, 567, L129
Laycock, S., Corbet, R. H. D., Coe, M. J., et al. 2005, ApJS, 161, 96

Lomb, N. R. 1976, Ap\&SS, 39, 447

Massey, P. 2002, ApJS, 141, 81

McGowan, K. E., Coe, M. J., Schurch, M. P. E., et al. 2008, MNRAS, 384, 821

Meyssonnier, N., \& Azzopardi, M. 1993, A\&AS, 102, 451

Negueruela, I. 1998, A\&A, 338, 505

Russell, S. C., \& Dopita, M. A. 1992, ApJ, 384, 508

Scargle, J. D. 1982, ApJ, 263, 835

Schmidtke, P. C., \& Cowley, A. P. 2005, AJ, 130, 2220

Schmidtke, P. C., Cowley, A. P., Levenson, L., \& Sweet, K. 2004, AJ, 127, 3388

Schwering, P. B. W., \& Israel, F. P. 1991, A\&A, 246, 231

Shtykovskiy, P., \& Gilfanov, M. 2005, MNRAS, 362, 879

Strüder, L., Briel, U., Dennerl, K., et al. 2001, A\&A, 365, L18

Szymanski, M. K. 2005, AcA, 55, 43

Turner, M. J. L., Abbey, A., Arnaud, M., et al. 2001, A\&A, 365, L27

Udalski, A., Kubiak, M., \& Szymanski, M. 1997, AcA, 47, 319

Udalski, A., Szymanski, M., Kubiak, M., et al. 1998, AcA, 48, 147

Ueno, M., Yokogawa, J., Imanishi, K., \& Koyama, K. 2000, PASJ, 52, L63

Wilms, J., Allen, A., \& McCray, R. 2000, ApJ, 542, 914

Yokogawa, J., Imanishi, K., Tsujimoto, M., Koyama, K., \& Nishiuchi, M. 2003, PASJ, 55, 161

Zaritsky, D., Harris, J., Thompson, I. B., Grebel, E. K., \& Massey, P. 2002, AJ, 123,855

Zavlin, V. E., Pavlov, G. G., Sanwal, D., \& Trümper, J. 2000, ApJ, 540, L25 